JONATHAN D. ROSEN*

Universidad Internacional de Florida (Miami, EE.UU.)

ROBERTO ZEPEDA MARTÍNEZ ${ }^{* *}$

Universidad Nacional Autónoma de México (México D.F., México)

\title{
La Guerra contra las Drogas y la Cooperación internacional: el caso de Colombia ${ }^{* * *}$
}

\author{
The Drug War and International Cooperation: \\ the Case of Colombia
}

\section{A Guerra contra as Drogas e a Cooperação internacional: o caso da Colômbia}

\footnotetext{
${ }^{*}$ Actualmente se desempeña como Investigador del Instituto Jack D. Gordon de Políticas Públicas de la Universidad Internacional de Florida. Anteriormente, fue Analista de Investigación en el Centro de Investigación Aplicada (ARC). El Dr. Rosen obtuvo su Maestría en Ciencia Política en la Universidad de Columbia y recibió el título de Doctor en Estudios Internacionales de la Universidad de Miami. Sus más recientes publicaciones incluyen: Jonathan D. Rosen, The Losing War: Plan Colombia and Beyond (Albany, NY: State University of New York Press, 2014); y Bruce M. Bagley and Jonathan D. Rosen, eds., Drug Trafficking, Organized Crime, and Violence in the Americas Today (Gainesville, FL: University Press of Florida, 2015). Correo electrónico: jonathanrosenrosen@gmail.com

** Doctor en Ciencias Políticas por la Universidad de Sheffield. Actualmente se desempeña como Investigador de tiempo completo en el Centro de Investigaciones Sobre América del Norte (CISAN) de la Universidad Nacional Autónoma de México (UNAM).Correo electrónico: zepeda_roberto@hotmail.com

*** Este artículo es producto del proyecto de investigación: La Guerra contra las drogas en América Latina y su impacto en la seguridad, UMAR (CUP:21EI14O2), desarrollado en el periodo enero 2014-septiembre 2015. Artículo de investigación recibido el 10/11/2014 y aprobado el 30/12-2015.
} 


\section{Cómo citar}

Rosen, J. D. y ZePedA MARTíneZ, R. (2016). La Guerra contra las Drogas y la Cooperación internacional: el caso de Colombia. Revista CS, no. 18, pp. 63-84. Cali, Colombia:

Facultad de Derecho y Ciencias Sociales, Universidad Icesi.

DOI: http://dx.doi.org/10.18046/recs.i18.1848 


\section{Resumen}

Abstract

Resumo

Este artículo analiza la política exterior de los Estados Unidos (EE.UU.) hacia Colombia con respecto al narcotráfico. El análisis se centra no solo en la Guerra contra las Drogas en Colombia sino también en los intereses de los EE.UU. en este país y en la región. La posición de Washington frente a este problema revela la política exterior de los EE.UU. hacia otros países en la región sobre el narcotráfico y el crimen organizado. Este trabajo analiza la cooperación entre los EE.UU. y Colombia en los esquemas señalados. Al final se hace un balance tanto del tipo de cooperación entre EE.UU. y Colombia, como a los resultados al tipo de cooperación.

PALABRAS CLAVE:

Colombia | Guerra contra las drogas | cooperación | Plan Colombia | Estados Unidos

This article examines the U.S. foreign policy towards Colombia with respect to drug trafficking. The analysis concentrates not only on the War on Drugs in Colombia but also on the interests of the U.S. in this country and in the region as a whole. The position of Colombia in the War on Drugs and the response of Washington to this problem highlight the foreign policy of the U.S. towards other countries in the region regarding drug trafficking and organized crime. This work analyzes cooperation between the U.S. and Colombia regarding the drug war. We conclude the article by assessing the cooperation between the United States and Colombia, taking into account the outcomes but also the kind of predominant cooperation..

KEYWORDS:

Colombia | War on Drugs | cooperation | Plan Colombia | United States

Este artigo analisa a política exterior dos Estados Unidos (EEUU.) com relação à Colômbia com respeito ao narcotráfico. A análise se foca não somente na Guerra contra as Drogas na Colômbia, mas, também nos interesses dos EEUU nesse país e na região. A posição de Washington diante deste problema revela a política exterior dos EEUU com relação aos outros 
países na região sobre o narcotráfico e o crime organizado. Este trabalho analisa a cooperação entre os EEUU. e a Colômbia nos esquemas indicados. Ao final se faz um balance do tipo de cooperação entre os EEUU. e a Colômbia, em quanto aos resultados, mas também em quanto ao tipo de cooperação.

\section{PALAVRAS CHAVES:}

Colômbia | Guerra contra as drogas | cooperação | Plano Colômbia | Estados Unidos 


\section{Introducción}

Desde la década de los 90 Colombia ha sido el principal productor y exportador de cocaína en el mundo. Una serie de condiciones estructurales subyacentes convierten a este país en el mejor lugar del continente americano para concentrar actividades de cultivo, manufactura y exportación de cocaína. Algunas de las causas son independientes entre sí, pero otras interactúan fortaleciendo su importancia. Estas condiciones estructurales subyacentes en Colombia son: la des-legitimización del régimen, la debilidad de la sociedad civil, la amplia propensión a resolver disputas por medio de la violencia, la geografía del país, la estructura del sistema político y de partidos, obstáculos para la movilidad social ascendente, la gran escala de actividades económicas ilegales, y la aceptación social del contrabando y lavado de dinero (Lee y Thoumi, 2003).

Estos factores dan cuenta de la importancia que el tráfico de drogas ha tenido para Colombia y países de la región andina; principalmente la cocaína, dirigida al mercado de América del Norte, pero que también involucra a países de tránsito en Centroamérica, el Caribe y México. La disputa por el control del cultivo, la producción y el tráfico de drogas ha generado violencia en Colombia: en las décadas de los 80 y los 90 la violencia alcanzó niveles alarmantes.

Durante el periodo 1980-200o, un gran número de periodistas, jueces, funcionarios de justicia y políticos fueron asesinados en Colombia porque eran vistos como obstáculos por los carteles del narcotráfico, principalmente los de Cali y Medellín. Asimismo, se ha demostrado un vínculo entre estos carteles y grupos guerrilleros en Colombia como las Fuerzas Armadas Revolucionarias de Colombia (FARC) y el Ejército de Liberación Nacional (ELN), contratados por los carteles para proteger los cultivos de enervantes. Los carteles colombianos también han estrechado vínculos con carteles mexicanos y de otros países de la región para introducir droga a Estados Unidos (Antonopoulos, 2008).

En el año 2000 se firmó el Plan Colombia entre Colombia y Estados Unidos, un tratado de cooperación internacional destinado a combatir y erradicar el cultivo y tráfico de drogas que surge de la región andina. Este artículo analiza el contexto en el que se negoció y gestó el Plan Colombia, así como sus logros, resultados y la experiencia que se puede extraer de la forma de cooperación entre estos países en otros escenarios similares.

\section{La Guerra contra las Drogas de Estados Unidos en Colombia}

No se puede entender la Guerra contra las Drogas y la política exterior de los EE.UU. y sus consecuencias en muchos países de América Latina, sin un contexto político e histórico (Bagley, 2001), crucial para entender por qué muchos países tienen altos niveles 
de violencia en el siglo XXI. La Guerra contra las Drogas de los EE.UU. inició en 1971 con la declaración del Presidente Richard Nixon. Sin embargo, vale la pena mencionar que Nixon reconoció un aspecto de la Guerra contra las Drogas que sus sucesores omitieron. Nixon identificó el problema correctamente: el tráfico de drogas existe porque hay consumidores y un amplio mercado, e invirtió recursos para combatir el consumo (Bagley, 2013a y 2013b; Cuellar, 2008; Carpenter, 2003).

La fase moderna de la Guerra contra las Drogas empezó con el Presidente Ronald Reagan en los 8o. Reagan consideró que la producción y el tráfico de las drogas ilegales, particularmente en la región andina, representaban una grave amenaza a la seguridad de los EE.UU. Por lo tanto, inició un proceso de securitización ${ }^{1}$ de la Guerra contra las Drogas, y convirtió esta guerra en el primer objetivo y prioridad de la seguridad nacional de su administración. Como resultado, Reagan implementó algunos programas importantes, como Plan Dignidad y Operation Blast Furnance (Bagley, 2012: 3-4), para combatir la producción de drogas en los países andinos.

La Guerra contra las Drogas durante la Administración Reagan evidenció los objetivos de Washington y su política exterior hacia América Latina. El primer objetivo de la política exterior de los EE.UU. siempre ha sido la seguridad. Reagan persuadió a la población de los EE.UU. de que el narcotráfico representaba una gran amenaza y que podría destruir las vidas de muchas personas y, sobre todo, afectar a los jóvenes. Muchas personas en los EE.UU. han visto los efectos que drogas como el "crack" -un derivado de la cocaína- han tenido en ciudades como Nueva York o Chicago. Reagan securitizó la Guerra contra las Drogas porque atemorizó a la sociedad y la convenció de que esta batalla era necesaria. En particular, la clase media representó -y continúa representando-un aliado formidable para quienes propugnan la necesidad de la Guerra contra las Drogas porque no quieren que sus hijos tengan problemas con ellas.

Durante los 8o, la Administración Reagan se concentró en la oferta y el suministro de drogas (Carpenter, 2003), pero descuidó la demanda. Reagan consideraba que los EE.UU. tenían que combatir la producción de las drogas. El 100 \% del cultivo de hojas de coca durante esta época era producido en Perú, Bolivia, y Colombia. Como consecuencia de las “victorias parciales" (Bagley, 2013a:3-5) de la Guerra contra las Drogas, la producción de coca se trasladó a Colombia. Para el año 200o, Colombia se convirtió en el primer cultivador de hojas de coca, así como productor y traficante de cocaína en todo el mundo. En suma, aunque se han modificado los lugares del cultivo y tráfico de cocaína, los países involucrados siguen siendo Colombia, Perú y Bolivia. Cuando un gobierno trata de

1. Securitización es un proceso que consiste en otorgarle prioridad a un tema o asunto colocándolo como la mayor amenaza a la seguridad de un país. Los académicos pueden estudiar el proceso de securitización mediante el análisis de discursos y examinando los recursos financieros dirigidos a ciertos programas. Ronald Reagan aplicó exitosamente la securitización de las drogas convenciendo a la población americana de que el tráfico de drogas representaba una amenaza grave a la seguridad nacional de los Estados Unidos. Una explicación más detallada sobre el concepto: Buzan, Weaver y Wilde (1998). 
erradicar el cultivo y combatir la producción de drogas, los lugares cambian para impedir la intervención de los cuerpos de seguridad (Bagley, 2013a:3-4; 2013b).

Evidentemente, la Administración Reagan también “cooperó" con otros países de la región andina, particularmente con Perú y Bolivia. Sin embargo, estos países andinos no eran aliados similares, y los EE.UU. usaron su poder y dictaron los términos y las condiciones de la asistencia. En otras palabras, los EE.UU. usaron su hegemonía para influir en otros países en la región (Bonilla, 2006). Bolivia y Perú eran países con mayores rezagos económicos y con un nivel de desarrollo menor al de Colombia, y necesitaban la cooperación y asistencia de los EE.UU. para combatir el cultivo de coca; y estos países se sometieron a los designios de los EE.UU.

\section{La Guerra contra las Drogas y el Presidente Clinton}

La estrategia de los EE.UU. y su Guerra contra las Drogas continuaron durante la presidencia de William Clinton (1993-200o), a pesar de pertenecer al Partido Demócrata. El caso de Colombia, particularmente la implementación del Plan Colombia, es un ejemplo crucial para entender la cooperación entre los EE.UU. y otros países en la Guerra contra las Drogas. William Clinton, como su predecesor Ronald Reagan, consideró al narcotráfico en Colombia como una amenaza que podría afectar a la seguridad de los EE.UU. Colombia posee una posición geográfica estratégica y es un país importante en la región andina. Los grandes problemas de seguridad de Colombia pueden impactar el comercio y la economía en la región. Los EE.UU. y Colombia tuvieron relaciones ríspidas durante el gobierno de Clinton y su homólogo en Colombia, Ernesto Samper (1994-1998). Samper nunca fue un aliado incondicional para los EE.UU., y las relaciones entre los países se deterioraron rápidamente. La Administración Clinton percibía al Presidente Samper como poco comprometido en el combate a las drogas, por lo que descertificó a Colombia por tres años consecutivos. Este es un ejemplo de la propensión de EE.UU. a usar su poder hegemónico en la región y dictar las condiciones a otros países de forma unilateral. EE.UU. sancionó a Colombia por su insubordinación y su falta de voluntad para obedecer las reglas y los dictados de la política exterior de EE.UU. (Crandall, 2002: 101-110).

El sucesor de Ernesto Samper en la presidencia de Colombia, Andrés Pastrana (1998-2002), se convirtió en un aliado con más potencial para los EE.UU. La Administración Clinton pensó que tenía una oportunidad para mejorar las relaciones con Colombia. Clinton invitó a Pastrana a la Casa Blanca para mejorar las relaciones con el nuevo Presidente de Colombia. Sin embargo, una vez más, se hizo evidente que Pastrana y Washington no eran aliados en una posición de igualdad (Crandall, 101). Hacia finales de la década de 1990, Colombia necesitaba asistencia y ayuda de los EE.UU. porque tenía muchos problemas sociales, económicos y de inseguridad. Colombia 
seguía siendo uno de los países más inseguros del mundo, a pesar de la destrucción de los cárteles más poderosos: el Cartel de Cali y el Cartel de Medellín (Crandall, 78-84). La consecuencia de la destrucción de los carteles fue la fragmentación y la creación de organizaciones más pequeñas, o "cartelitos," que se disputaban el control de la producción y tráfico de drogas.

El Presidente Pastrana reconoció que Colombia necesitaba ayuda de los EE.UU. porque no podía solucionar los problemas por sí mismo. En 1998, quería lograr la paz en el país y trató de negociar con las guerrillas en Colombia para resolver un conflicto interno de varias décadas. Pastrana negoció con el grupo guerrillero más poderoso, Las Fuerzas Armadas Revolucionarias de Colombia, y otorgó una zona de despeje, que comprendía un territorio equivalente al tamaño de Suiza. Esta zona despeje resultó un desastre, ya que las FARC se movieron por esta zona con total libertad para realizar sus operaciones, incluyendo el tráfico de drogas (Rosen 2014).

Después del fracaso de la zona de despeje, Pastrana habló con Clinton para solicitar ayuda. La Administración Clinton contestó a Pastrana que su gobierno tenía que formular una iniciativa de cooperación con los EE.UU. Pastrana escribió un plan conocido como el Plan Colombia; su versión se enfocaba en el proceso de paz, en la economía y el desarrollo. Para Pastrana, no se podía separar el tema de las drogas del conflicto interno porque las FARC traficaron -y siguen traficando- drogas, que representan un gran porcentaje de su financiación. La versión del Plan Colombia de Pastrana se enfocó menos en la Guerra contra las Drogas y más en los problemas subyacentes como el conflicto interno y el desarrollo. Pastrana redactó un Plan que implicaba la asistencia de muchos países, particularmente países de Europa, con un costo de 7,5 mil millones de dólares.

Como resultado de la influencia y persuasión del Zar de las Drogas (Drug Czar) en el periodo 1996-2001, Barry McCaffrey, la Administración Clinton usó su poder para cambiar la estrategia del Plan Colombia. Clinton reconoció que los EE.UU. no podían intervenir en el conflicto interno de Colombia porque no querían que se repitiera una situación como la de Vietnam. Entonces, los EE.UU. usaron su poder para reorientar la estrategia del Plan Colombia. El primer objetivo del Plan Colombia para la Administración Clinton era el combate a las drogas. Colombia representaba una amenaza a la seguridad de la región y de los EE.UU. por las actividades de los narcotraficantes. En suma, la Guerra contra las Drogas fue uno de los temas más importantes para la Administración Clinton. El Congreso de los EE.UU. aprobó el Plan Colombia, y el Presidente Clinton lo firmó en Julio del año 200o. En ese mismo año, Colombia recibió más recursos que todos los países en el hemisferio y en el mundo, excepto Israel y Egipto (Crandall: 2002, 1)². 
La versión final del Plan Colombia, no obstante, resultó muy diferente de la versión que Pastrana escribió originalmente. La comunidad internacional no estaba de acuerdo con la nueva versión de los EE.UU. del Plan Colombia y se rehusó a invertir recursos en esa iniciativa. De esta manera, los EE.UU. invirtieron la totalidad de los recursos del Plan Colombia. Es importante entender cómo la política interna de los EE.UU. impactó en la formulación del Plan Colombia. Clinton consideraba que tenía que demostrar poder en la Guerra contra las Drogas, particularmente después de que los Republicanos tomaron el poder del Congreso. Por esto, el Plan Colombia representó una oportunidad para que Clinton demostrara que era implacable en la lucha contra las drogas. En otras palabras, el Plan Colombia sirvió como un mecanismo para silenciar a los críticos de Clinton.

\section{Lecciones del Plan Colombia}

¿Cuáles son las lecciones de la formulación e implementación del Plan Colombia respecto a la política exterior de los EE.UU. y a la Guerra contra las Drogas? Washington, generalmente pensaba -y piensa hoy-que entiende la situación en América Latina y puede resolver los problemas de los países en la región. Otros líderes y expertos de América Latina han criticado a los EE.UU. y su Guerra contra Las Drogas, argumentando que la estrategia ha fracasado (Bagley y Tokatlian, 1992, 1985; Bagley, 1988), y que Washington tiene que cambiarla. Expertos como Thoumi (1995: 205) y Carpenter (2003: 22) han observado que líderes de América Latina argumentan que el problema no es la producción y el suministro de las drogas en la región, sino el consumo de drogas en los EE.UU. Washington, sin embargo, siempre ha usado su poder e influencia para determinar la agenda en la relación con América Latina. En realidad, los EE.UU. se han comportado de acuerdo a los parámetros de los realistas (es decir, de la teoría del realismo en las RR.II.). Lo más importante para los realistas, y los EE.UU., es el concepto de seguridad en un mundo caracterizado como anárquico. Los EE.UU. tienen más recursos y poder que todos los países de la región juntos y pueden determinar o cambiar la agenda de acuerdo a sus intereses. Para 1999, Colombia había visto muchos obstáculos, y Pastrana entendió que necesitaba ayuda de EE.UU. para impedir un desastre en Colombia; aceptó la reorientación del Plan Colombia porque necesitaba los recursos (Rosen 2014).

Una de las lecciones del Plan Colombia sobre la política exterior de EE.UU. en la región andina es que EE.UU. tradicionalmente no trabaja con otros países ni instituciones para desarrollar estrategias bilaterales. El Plan Colombia fue una iniciativa de los "gringos" del norte porque otros países no estaban de acuerdo. Si Washington hubiera trabajado con otros países, habría tenido una versión del Plan Colombia muy diferente. La realidad es que EE.UU. no ha trabajado, ni negociado con otros países 
porque piensan que no es necesario. Los EE.UU. implementaron su visión del Plan Colombia, un plan que invirtió el 80\% del financiamiento en el ejército y otros componentes hard, como tecnología para el ejército.

En suma, el Plan Colombia demuestra que los EE.UU. estaban más preocupados con la seguridad nacional que con su reputación en la región: su primer objetivo siempre fue -y sigue siendo-la seguridad. Washington siempre ha tenido sus concepciones sobre la Guerra contra las Drogas y sobre la estrategia más adecuada. En general, los EE.UU. no se han preocupado por la cooperación con otros países. En vez de eso, se han enfocado en la resolución del problema de las drogas de acuerdo a sus intereses. En el caso de Colombia, el Presidente Clinton y su administración comunicaron al presidente Pastrana que el problema eran las drogas y la solución pasaba por combatir el cultivo, la producción y el tráfico de las mismas. Evidentemente, es necesario mencionar que Colombia y EE.UU. cooperaron; ya que Colombia recibió recursos estadounidenses para implementar las estrategias diseñadas y tuvo que cooperar con Washington (Rosen, 2014).

\section{El 11 de septiembre, Bush y Uribe}

No hubo solamente un Plan Colombia; este tuvo diferentes fases y sus objetivos cambiaron durante su implementación. Los eventos trágicos del 11 de septiembre de 2001 redefinieron los objetivos y prioridades de la política exterior de EE.UU. La prioridad de la administración de George W. Bush fue la necesidad de combatir el terrorismo en todo el planeta. Bush empezó una Guerra contra el Terrorismo y dijo que los EE.UU. tenían que combatir a los terroristas (Bush, 2001), así, la Guerra contra las Drogas se subordinó a la Guerra contra el Terrorismo.

El sucesor de Pastrana, Álvaro Uribe (2002-2010), prometió combatir a las FARC y reconoció que Colombia necesitaba ayuda de los EE.UU. para mejorar en seguridad. Algunos expertos dijeron que Colombia era un país extremadamente peligroso y hablaron sobre un Estado a punto de fracasar. Uribe aprendió de la historia de Colombia, particularmente durante la administración de Pastrana, y se dio cuenta de que tenía que modificar la estrategia del Plan Colombia. Como se ha mencionado, el primer objetivo de la Administración Bush fue la Guerra contra el Terrorismo; entonces, Uribe cambió la percepción del conflicto en Colombia para describir el conflicto interno colombiano como un problema de terrorismo, concretamente definió a las FARC como narco-terroristas (Chernick, 2012). De esta manera, las narco-guerrillas terroristas trafican drogas y son una amenaza a la estabilidad y la seguridad de Colombia. Este movimiento fue muy inteligente porque Uribe sabía que la política exterior de los EE.UU. había cambiado y Colombia tuvo que reorientar su agenda. Colombia representó una oportunidad para el Presidente Bush de demostrar que no quería pelear con el mundo musulmán. Mediante 
su apoyo a Colombia Bush pudo responder a los críticos que le acusaban de enfrentarse al mundo musulmán y demostrar que los EE.UU. apoyaron a todos los países a combatir el terrorismo independientemente de la religión del país (Rosen 2014).

Los presidentes Bush y Uribe fueron aliados afines porque coincidían en el combate al terrorismo. A finales de la administración Bush, Uribe era el único aliado de EE.UU. en la región (Kraul, 2008). Bush le recompensó con más ayuda; sin embargo, es importante mencionar que la administración Bush apoyó y aceptó la reorientación del Plan Colombia porque EE.UU. estaba de acuerdo con la estrategia. Si no lo hubieran estado, la administración Bush la habría cambiado. Uribe tuvo éxito en reformular el Plan Colombia y reorientar la historia de su país. En realidad, Uribe alteró la historia de su propio país con la finalidad de reformar el Plan Colombia. La Administración Bush apoyó a Colombia no solamente en cuestiones de seguridad; los dos países tienen una relación comercial importante y Colombia una larga historia democrática, lo que coincide con los países que apoya el Gobierno estadounidense. El Plan Colombia se transformó por iniciativa bilateral.

Bush, al igual que sus predecesores, no utilizó instituciones ni buscó cooperación con otros países. Al contrario, tenía una visión propia sobre cómo resolver el problema. EE.UU. utilizó su poder para combatir el terrorismo en Colombia y la Administración Bush lo hizo sin colaborar con otras instituciones ni pedir permiso a nadie: para los neoconservadores, EE.UU. es el país más poderoso y tiene la responsabilidad de intervenir en otros países. La comunidad internacional pudo corroborarlo cuando EE.UU. impuso en la ONU su resolución sobre Iraq. Tenemos que cuestionar el diseño de la ONU. Hay cinco países que son miembros permanentes del Consejo de Seguridad de la ONU: ellos tienen todo el poder; son los cinco países que ganaron la Segunda Guerra Mundial: EE.UU., China, Rusia, Gran Bretaña, y Francia. A pesar de que la ONU tiene 193 países miembros (United Nations, 2013), estos cinco concentran el poder. Por ello, la administración Bush consideró que no tenía que pedir permiso en la Guerra contra el Terrorismo y en la Guerra contra las Drogas. John Mearsheimer, en The False Promises of International Institutions (1994), establece que los países poderosos pueden dominar las instituciones y no tienen que escuchar a otros países. En suma, las administraciones de George W. Bush y Álvaro Uribe tuvieron las mismas visiones y Colombia cambió su estrategia para cumplir los requisitos de EE.UU. y recibir mayor financiamiento.

\section{La cooperación internacional}

Durante su presidencia, Uribe no priorizó las relaciones con sus vecinos. Acusó a los presidentes de Ecuador y Venezuela de apoyar a las FARC (Refshauge, 2012). Obviamente, estas afirmaciones no mejoraron el soft power (Nye, 2004) de Colombia ni estimularon 
la cooperación entre los países de la región. Como la de Uribe, la administración Bush tuvo relaciones difíciles con otros países de la región en esos años. Se puede caracterizar la doctrina de política exterior adoptada por Bush como de realismo neoconservador (Nuruzzaman 2006): la Administración Bush pensó que tenía la responsabilidad de ayudar e intervenir en otros países y trató de cambiar la imagen de EE.UU. en el mundo. Durante esa administración aumentó la política unilateral. Bush apoyó Colombia, mas no con recursos para instituciones, educación y prevención: el 80\% de los recursos se destinaron al Ejército y a programas de fumigación. Estos programas no resolvieron los problemas subyacentes. Sin embargo, las administraciones de Bush y Uribe argumentaron que el Plan Colombia era un modelo para otros países con problemas de narcotráfico y terrorismo (Isacson, 2002).

De hecho, la Administración Bush designó un plan similar para México: el Plan México (Lendam, 2008), que posteriormente cambió nombre a Iniciativa Mérida (IM), que costó 1,4 mil millones de dólares (Chabat, 2010); mientras que el Plan Colombia costó 8 mil millones de dólares entre 2000-2012 (Bagley 2012a). La idea de que un mismo modelo sea válido para cualquier país presenta varios problemas (Isacson, 2002), en primer lugar, México y Colombia tienen historia, cultura e instituciones diferentes; además son problemas diferentes. Benítez Manaut (2014) identifica una serie de diferencias entre México y Colombia, entre las que destacan:

- Los gobiernos de Colombia y EE.UU. definieron la actividad criminal en Colombia como una síntesis del tráfico de drogas y el terrorismo: narcoterrorismo. Esto condujo a programas asistenciales basados en estrategias “antiterrorismo”. Mientras en México, antes del ataque al casino Royale en Agosto de 2011, las autoridades no habían vinculado la actividad criminal de los carteles con el terrorismo, ni habían definido sus políticas como "antiterroristas". Los carteles de la droga eran vistos simplemente como "crimen organizado".

- Como país productor, el problema de Colombia está enraizado en el área rural. Mientras que en México, en donde la cocaína es almacenada, es un fenómeno urbano.

- En Colombia, varios frentes del grupo armado revolucionario FARC se unieron a las fuerzas criminales, permitiendo que la lucha contra ellos se definiera como guerra contra el terrorismo. Mientras que en México no hay movimientos armados vinculados al tráfico de drogas.

- En Colombia no hay un "nacionalismo militar" antiestadounidense, como existe en México; por ello, la cooperación entre Estados Unidos y México se debe dar de manera cuidadosa.

Es lamentable que se argumente que el Plan Colombia es un modelo, pues no tuvo éxito en contra del narcotráfico. Sin embargo, EE.UU. continúa usando su poder para diseñar la agenda de cooperación. El gobierno de México aceptó la IM porque necesitaba asistencia de EE.UU. Pero en el siglo XXI México tiene problemas con la violencia y el crimen organizado a causa de los EE.UU. y su fallida guerra contras las drogas (Bagley, 2O12b). 
El problema con la política exterior y la cooperación de EE.UU. es que no se ha aprendido de los fracasos pasados: las rutas de las drogas vuelven a Colombia y otros países en América Central y el Caribe. Muchos expertos han analizado la política exterior de EE.UU. y el narcotráfico, pero durante la administración Bush el gobierno de EE.UU. no escuchó las críticas al respecto. La primera lección de la Guerra contra las Drogas es que las rutas siempre van a cambiar porque los narcotraficantes se adaptan para sobrevivir. EE.UU. continúa apoyando a otros países a través de una estrategia de militarización, aunque estos programas no resuelven los problemas subyacentes como la falta de educación, problemas socioeconómicos, o el consumo y la demanda.

\begin{tabular}{|c|l|}
\hline GRÁFICA 1 & $\begin{array}{l}\text { Cultivo global de hoja de Coca en Bolivia, Perú y Colombia, } \\
\text { 1990-2011 (hectáreas) }\end{array}$ \\
\hline
\end{tabular}

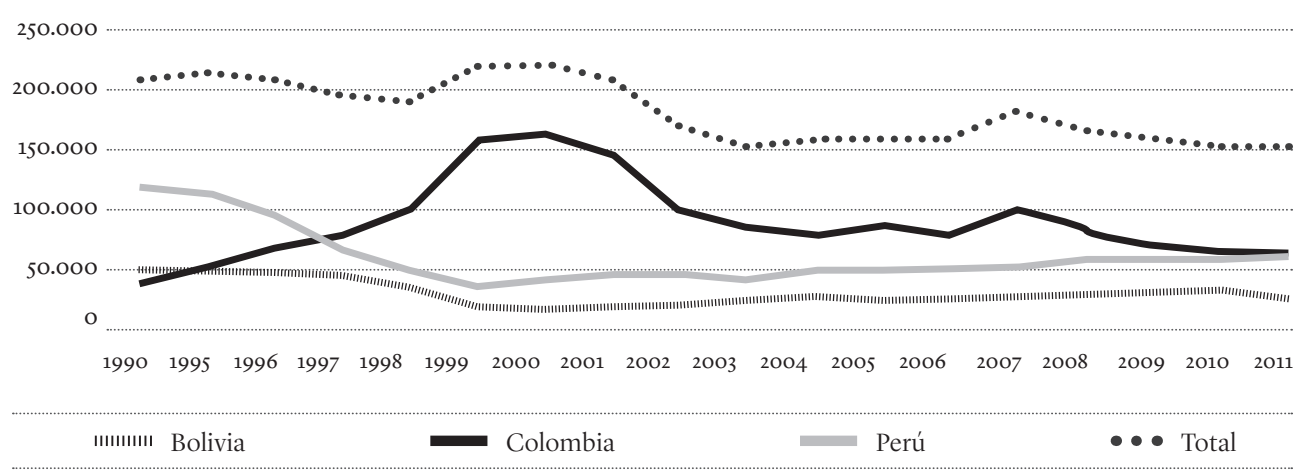

Fuente: Preparada por los autores con datos de UNODC, 2010 y 2013

\section{GRÁFICA 2 Producción de Cocaína, decomisos y oferta en los mercados, 1990-2008}

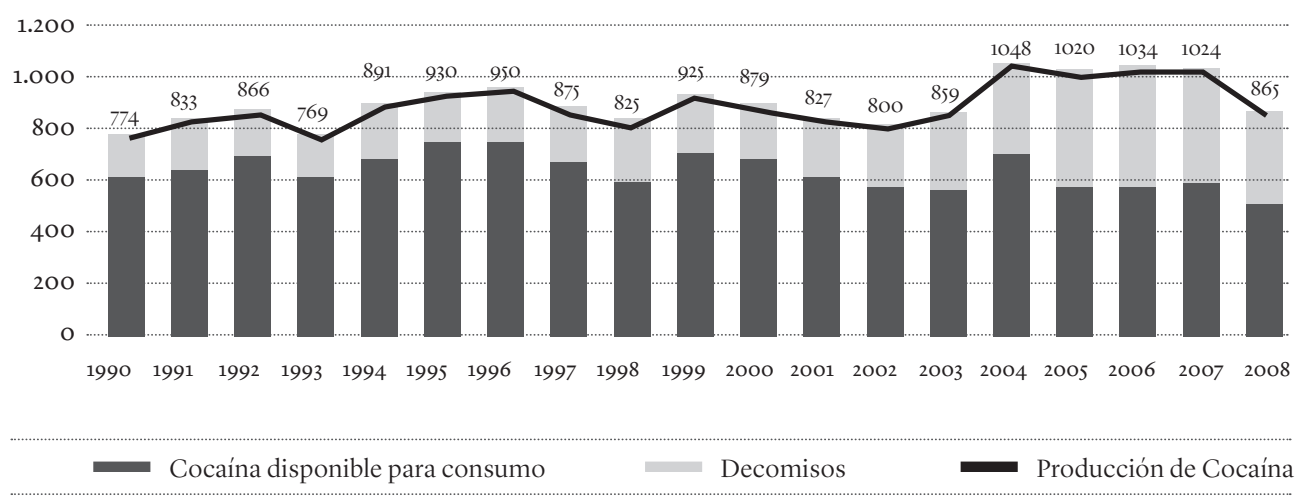


MAPA 1 | Principales flujos globales de cocaína

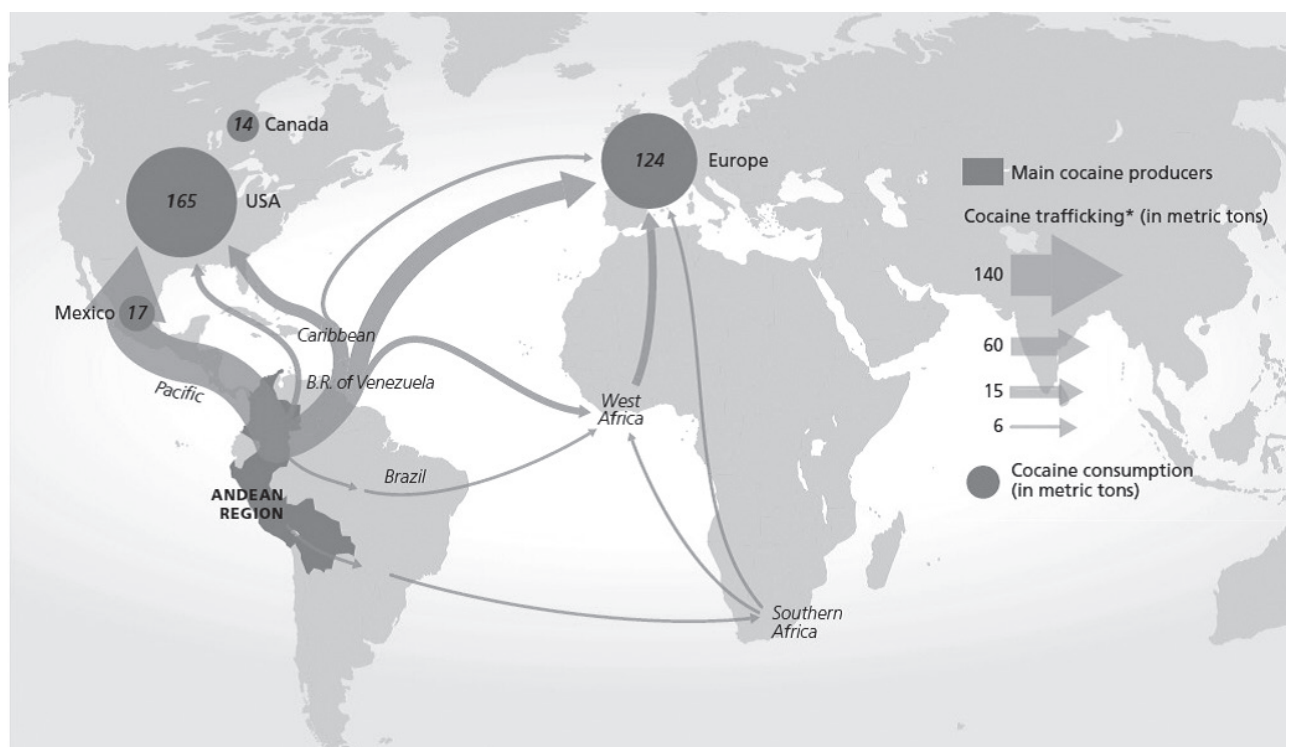

Fuente: UNODC, 2008, citado por Bagley, 2012a.

A pesar de los éxitos sustanciales del Plan Colombia y de las políticas de "Seguridad Democrática" implementadas por el presidente Uribe, para el año 2010 Colombia siguió siendo el principal productor de hoja de coca y cocaína refinada en los Andes, y la violencia relacionada con las drogas y el crimen aumentó de nueva cuenta. El reporte sobre las drogas de la UNODC (2011) establece que el área usada para cultivar la coca en Colombia decreció alrededor del 15\% en 2010, dejando a Colombia ligeramente por encima de Perú como el mayor productor de hoja de coca en el mundo. En 2011, el área de cultivo de coca en Colombia aumentó en 3\%, aunque de acuerdo a estadísticas compiladas por el gobierno de Estados Unidos hubo un declive. El área cultivada en Colombia abarcaba 62.0oo hectáreas, en comparación con las 73.0oo reportadas en 2009. Como consecuencia no deseada de la Guerra contra las Drogas promovida por Estados Unidos en Colombia, el centro neurálgico de los grupos criminales del tráfico de cocaína cambió gradualmente de Colombia a México (Bagley, 2012a; UNODC, 2011).

Aunque la administración Uribe y el Plan Colombia apoyado por Estados Unidos triunfaron al menos parcialmente en Colombia en la guerra contra la cocaína, las principales redes de drogas en México aprovecharon el vacío dejado para controlar el tráfico de cocaína desde Colombia. Consecuentemente, la violencia relacionada con el narcotráfico y el crimen organizado se desplazó hacia el norte, a territorio mexicano. Varias organizaciones de traficantes mexicanos se disputaron este altamente lucrativo comercio ilegal hacia el gran mercado estadounidense (Bagley, 2012a). 
Es común escuchar que el tráfico de drogas en Colombia prácticamente se ha erradicado. Sin embargo, diversas evidencias sugieren que la situación es muy distinta: el narcotráfico no ha desaparecido, su estructura regional en el hemisferio occidental se ha transformado radicalmente (Garay y Salcedo, 2012). El menor número de víctimas relacionadas con el narcotráfico y la detención o eliminación de capos notorios, implica una reconfiguración de los vínculos entre grupos ilegales, de tal suerte que siguen operando pero aliados ahora con los cárteles mexicanos y guatemaltecos (Bagley, 2012a).

UNODC considera que el valor total de venta de cocaína a nivel mundial en el año 2009 fue de aproximadamente US\$85.00o millones (OEA, 2013). Esta misma fuente estima que los principales mercados de la cocaína son Norteamérica (US\$40.ooo millones, $47 \%$ del mercado mundial), seguido de Europa Occidental y Central (US\$34.ooo millones, 39\% del mercado mundial), mientras que América Latina consume cerca del $4 \%$. La producción mundial de cocaína, que disminuyó entre 2000 y 2008 , se ha estabilizado en alrededor de 800 toneladas métricas por año. Colombia, que anteriormente producía la mayor cantidad de cocaína, disminuyó su producción durante la última década, al tiempo que en Perú y Bolivia aumentaba (UNODC, 2013; OEA, 2013).

\section{La Doctrina Obama}

La elección del Presidente Obama en 2008 representó una oportunidad para cambiar la estrategia de la Guerra contra las Drogas: se requerían nuevas estrategias y políticas. La administración Obama sabe que EE.UU. no puede seguir siendo el policía del mundo ni continuar con la guerra global contra el terrorismo. El mejor ejemplo de la doctrina Obama es el caso de Libia: la administración Obama trabajó con otros países y organismos internacionales como la ONU para resolver el problema. Obama se ha dado cuenta de que EE.UU. no puede hacer todo y tiene que trabajar y cooperar con otros. Obviamente, EE.UU. apoyó la solución al conflicto de Libia (Elliot, 2011) a través de recursos financieros. Sin embargo, otros países, como Francia, también fueron importantes en las negociaciones y en la ejecución del plan. Asimismo, otras instituciones como la ONU y la OTAN fueron centrales en la misión y la estrategia. A pesar de que la administración Obama es más propensa a recurrir a las instituciones, ha sido decepcionante con respecto a la Guerra contra las Drogas: ha atacado lugares que distribuyen marihuana medicinal. Nick Wing y Luke Johnson revelan que "un análisis del grupo a favor de la marihuana para usos medicinales, Americans for Safe Access (ASA), encontró que la Administración Obama ha gastado casi 300 millones de dólares en el combate a la marihuana medicinal en estados que ya han legalizado esta droga" (2013:1).

El reporte calcula que la cantidad total del gasto federal en la intervención de la marihuana medicinal equivale a 289 millones de dólares durante un periodo de 4 años y 
medio del gobierno de Obama. De acuerdo a este análisis, solamente la DEA gastó 4 por ciento de su presupuesto en $2 \mathrm{O} 11$ y 2012 combatiendo la marihuana medicinal en 20 estados. Considerando el total, la participación de Obama representa la mayoría de lo que ASA dice que son 483 millones de la guerra de demandas, acusaciones, e intentos de incautaciones de bienes emprendidos por el Departamento de Justicia bajo los tres presidentes anteriores (Wing y Johnson, 2013: 1).

El gobierno federal decide procesar tiendas de marihuana medicinal en diferentes estados de la unión americana, lo que representa un desperdicio de dinero y demuestra que el desempeño de la administración Obama en la lucha contra las drogas ha sido decepcionante. El presidente Barack Obama no contempla cambiar la clasificación de la marihuana como droga peligrosa, a pesar del creciente número de estados que han autorizado su uso con fines médicos. El presidente y su administración creen que perseguir a los usuarios de marihuana, especialmente a aquellos con padecimientos serios, así como a las personas que los cuidan, no es la mejor manera de usar los recursos policiales, de acuerdo el vocero presidencial de la Casa Blanca Josh Earnest (Notimex, 2013). La única medida positiva que Obama ha hecho respecto a la Guerra contra las Drogas fue rechazar las sentencias mínimas obligatorias que provienen de la administración Reagan. Esta ley condenaba a consumidores de "crack" a prisión por muchos años. La realidad es que el "crack" es más barato que la cocaína y ha devastado a comunidades pobres y minorías de los EE.UU.

\section{Perspectivas de cooperación}

Como se ha mencionado, Obama no cambió las tácticas fallidas en la Guerra contra las Drogas. La administración Obama, sin embargo, ha tratado de mejorar su reputación o soft power (Nye, 2004) en la región. La solución a los problemas del narcotráfico demanda cooperación entre los países en la región, y Obama se ha dado cuenta de que no puede intimidar a otros países con el poder de EE.UU; no ha confrontado ni implementado nuevas estrategias, argumentando que la legalización de las drogas no es la respuesta, pues impulsaría su comercio masivo, y sin límites las condiciones podrían ser más dañinas que las actuales (Agencia EFE, 2013). Los problemas derivados de la producción, tráfico y consumo drogas no tienen una "solución mágica” (Bagley, 2012b). Hay muchos problemas subyacentes como delincuencia, economía y desempleo, entre otros. Por lo tanto los países que consumen y los que producen drogas deben colaborar de manera coordinada para encontrar una solución. Muchos expertos han expresado que las estrategias en América Latina tienen que cambiar. Ethan Nadelmann lo explica de la siguiente manera: 
En 2009, los ex-presidentes Fernando Henrique Cardoso (Brasil), César Gaviria (Colombia) y Ernesto Zedillo (México) se reunieron con otros miembros de la Comisión Latinoamericana sobre las Drogas y la Democracia y concluyeron que había llegado el momento para romper el tabú y explorar alternativas ante el fracaso de la Guerra contra las drogas. En 2011, estos expresidentes se reunieron con el anterior Secretario General de las Naciones Unidas Kofi Annan, el anterior Secretario de Estado de los Estados Unidos George Shultz, el anterior director de la Reserva Federal de Estados Unidos Paul Volcker, el expresidente Suizo Ruth Dreifuss y otros miembros de la Comisión Global de Políticas de Drogas y convocaron a reformas fundamentales a las políticas de drogas nacionales y globales. Los expresidentes Jimmy Carter, Ricardo Lagos (Chile), Vicente Fox (México) y Aleksander Kwasniewski (Polonia) estaban entre los que apoyaban sus recomendaciones (Nadelmann, 2013: 1-2).

El reporte reciente de la Organización de Estados Americanos-OEA-representa un avance en la solución al tema de las drogas porque algunos países han reconocido que la Guerra contra las Drogas ha sido un fracaso y que los países tienen que cambiar la estrategia hasta ahora implementada. "El paradigma de 'Guerra contra las Drogas' que durante décadas fue defendido por Estados Unidos como la estrategia indicada para el combate del narcotráfico en la región está en crisis. Así quedó demostrado en la última Asamblea General de la OEA, en la que por primera vez se cuestionó la postura norteamericana y se debatieron alternativas de políticas antinarcóticos" (Barassi, 2013). La estrategia solamente puede cambiar con cooperación y coordinación; sin embargo EE.UU. y México no están a favor del informe. Esto es muy problemático por varias razones: primero, un país puede legalizar las drogas porque tiene soberanía. Habiendo dicho esto, hay que recordar la historia en Colombia. Es posible que EE.UU. descertifique un país que quiera legalizar las drogas. Aunque lo más probable es que Obama no vaya a hacer nada si se legalizaran las drogas en otros países, el principal punto es que esta no es una buena estrategia.

Es importante recordar que el crimen organizado y el narcotráfico no se circunscriben al contexto nacional sino que se enmarcan en el ámbito transnacional. En este sentido, un país no es el único responsable ni puede resolver los problemas relacionados con estos fenómenos sin apoyo externo. El problema del narcotráfico es complejo y los países necesitan debatir y cooperar no solamente sobre la legalización de la marihuana sino también sobre los problemas subyacentes, tales como instituciones débiles, corrupción, impunidad, educación, demanda de drogas, prevención, entre otros. Los países que producen, trafican y consumen drogas no pueden resolver los problemas sin esfuerzos honestos en colaboración y coordinación.

De manera interesante, Colombia ha promovido la exportación de su modelo de lucha contra el narcotráfico a otros países, aunque sea considerado un modelo fracasado. El expresidente de Colombia Álvaro Uribe ha promovido su modelo en 
otros países con problemas similares como México y Afganistán (Isacson, 2010). Como hemos visto en este artículo, imponer un modelo genérico de la lucha contra el narcotráfico en países diferentes no es lo más acertado. Sin embargo, el sucesor de Uribe en la presidencia de Colombia, Juan Manuel Santos, ha argumentado que otros países pueden aprender de las lecciones de Colombia, particularmente porque muchas personas se han visto afectadas.

\section{Discusión}

Este artículo ha examinado la cooperación de EE.UU. y Colombia en el contexto de la Guerra contra las Drogas, en especial el Plan Colombia, para entender la cooperación internacional en el combate a las drogas. En este caso, EE.UU. utilizó su poder e influencia para dictar las condiciones de la cooperación, que eran la seguridad de la región y su país. Ronald Reagan hizo de la Guerra contra las Drogas el primer objetivo de su administración; argumentó que los EE.UU. tenían problemas porque países como Colombia producían y traficaban drogas. En este sentido, construyó las condiciones y lanzó la guerra contra las drogas en la región andina. Desafortunadamente, la política exterior de EE.UU. no cambió mucho después de su administración (Carpenter, 2003).

Diversos analistas han observado el fracaso de esta guerra. Juan Gabriel Tokatlian (2012) ha señalado que en la Guerra contra las Drogas prevalece la decisión político-burocrática, electoral e ideológica de continuar el curso de acción trazado a pesar de los fracasos y frustraciones. Si se conciben paliativos -como el desarrollo rural alternativo o la erradicación forzada de cultivos- es para hacer más eficaz la tarea punitiva y, en segundo término, contener el rechazo social que produce la coerción. El mensaje es que la Guerra contra las Drogas no se puede ni se debe detener.

Sin embargo, EE.UU. tiene que cambiar sus estrategias y trabajar con los demás países de la región. Durante la administración de George W. Bush escaseó la cooperación en Latinoamérica; con excepción del presidente colombiano Uribe, Bush no tuvo aliados en América Latina. El problema del narcotráfico no afecta solo a un país u otro, es un problema global. Para resolverlo los países deben trabajar juntos y cooperar. Obama representó una nueva oportunidad para cambiar la política exterior de Estados Unidos; pero negocia con otros países no reformar la política antinarcóticos. Ethan Nadelmann (2012) ha observado que el gobierno de gobierno de Obama inició con grandes promesas para los reformistas de la política antidrogas, pero en los años recientes ha sido difícil distinguir sus políticas antidrogas de las de sus predecesores en la Casa Blanca. Nadelmann puntualiza que aunque el presidente Obama ha reconocido que la legalización es un tema totalmente legítimo para debatir-primera vez que un presidente en funciones hace tal afirmación-, su administración ha aplicado medidas y acciones desalentadoras. 
La mitad de todos los arrestos en Estados Unidos (más de 850,000 americanos) han sido por uso de marihuana, solo en 2010; 88\% por posesión (Nadelmann, 2012).

Obama ha continuado con las políticas de sus predecesores y no está a favor de la legalización. La solución es que los países en la región trabajen de manera coordinada para resolver el problema; sin embargo, también deben reformar sus propias políticas antinarcóticos. Obviamente, EE.UU. tiene corresponsabilidad en la Guerra contra las Drogas. Los países en la región tienen que reformar sus instituciones domésticas y resolver los problemas subyacentes; por ejemplo, México y Colombia tienen graves problemas de corrupción interna e instituciones débiles; asimismo, tienen altos niveles de desempleo, pobreza y desigualdad, y el sistema de justicia es deficiente.

La Guerra contra las Drogas en Colombia representa un caso particular pero pertinente; demuestra la falta de cooperación entre los países que producen, trafican y consumen drogas en el continente americano. Es un ejemplo evidente de la falta de cooperación entre los países andinos, centroamericanos, norteamericanos y del caribe; demuestra que falta cooperación integral y efectiva en el combate al crimen organizado y al tráfico de drogas. La Guerra contra las Drogas en Colombia no tuvo éxito porque no hubo cooperación multilateral efectiva promovida y alentada por Estados Unidos. Se tienen que modificar los esquemas, el tipo y el alcance de la cooperación para que en el futuro próximo se pueda resolver el problema del cultivo, producción y tráfico de drogas en la región.

\section{Referencias}

AGENCIA EFE (2013), "La legalización de drogas no es la respuesta: Barack Obama”, El Tiempo, disponible en línea: <http://m.eltiempo.com/politica/discurso-de-barack-obama-en-la-clausura-de-foro-de-empresarios/11566401> Consultado en 20/10/2013.

ANTONOPOUlOS, G. A. (2008), “Colombian Drug Cartels”, en Shanty, F. y Mishra, P. P. (2008). Organized Crime: From Trafficking to Terrorism (Vol. 1). ABC-clio, pp. 455-456.

BAGLEY, B. M. (1998). “US Foreign Policy and the War on Drugs: Analysis of a Policy Failure,” Journal of Interamerican Studies and World Affairs 30 (2/3), Special Issue: Assessing the Americas' War on Drugs, 189-212.

BAGLEY, B. M. (2001). "Drug Trafficking, Political Violence, and U.S. Policy in Colombia in the 199os”, en Mama Coca. <www.mamacoca.org> Consultado en 3/10/2013.

BAGLEY, B. M. (2012a). "Drug Trafficking and Organized Crime in the Americas: Major Trends in the Twenty-First Century". Woodrow Wilson International Center for Scholars, Latin American Program. 
BAGLEY, B. M. (2012b) "Tráfico de drogas y crimen organizado en América Latina y el Caribe en el Siglo XXI: retos de la democracia, Encuentro Internacional Drogas, Usos, y Prevenciones", 16 - 18 de Mayo 2012. Quito, Ecuador.

BAGLEY, B. M. (2013a). "Drug Control Policies in the United States: What Works and What? Patterns, Prevalence, and Problems of Drug Use in the United States," en Bagley y Rosen (eds.) Drug Trafficking, Organized Crime, and Violence in the Americas: Key Trends in the 21st Century, Gainsville, Florida: University Press of Florida (en revision).

BAGLEY, B. M. (2013b). "Principales tendencias del siglo XXI en cuanto al crimen organizado, el narcotráfico y la democracia en la región”. Trans-Pasando Fronteras, (3), pp. 47-54. Disponible en línea: <http://dx.doi.org/10.18046/retf.i3.1623> Consultado en 29/12/2014.

BAGLEY, B. M. y TOKATLIAN, J. G. (1985). “Colombian Foreign Policy in the 1980s: The Search for Leverage," Journal of Interamerican Studies and World Affairs 27 (3), 27-62.

BAGLEY, B. M. y TOKATLIAN, J. G. (1992). "Dope and Dogma: Explaining the Failure of U.S.-Latin American Drug Policies," en Jonathan Hartlyn, Lars Schoultz, Augusto Varas (eds.) The United States and Latin America in the 199os: Beyond the Cold War. Chapel Hill: The University of North Carolina Press.

BARASSI, S. (2013). "Una nueva estrategia contra la droga”, Portal del Sur - Agencia Latinoamericana de Noticias, 12 de junio. Disponible en línea: <http://portaldelsur.info/2013/o6/una-nueva-estrategia-contra-la-droga/> Consultado en 29/07/2013.

BENÍTEZ, R. (2014), "Mexico-Colombia: US Assistance and the Fight against Organized Crime", Llorente, M. V., McDermott, J., Benitez-Manaut, R., de Rincón, M. L. R., \& Bailey, J. (Eds.). One Goal, Two Struggles: Confronting Crime and Violence in Mexico and Colombia. Woodrow Wilson Center for International Scholars, 47-69.

BONILlA, A. (2006). "U.S. Andean Policy, the Colombian Conflict, and Security in Ecuador," en Brian Loveman, ed. Addicted to Failure: U.S. Security Policy in Latin America and the Andean Region. Lanham, Maryland: Rowman \& Littlefield Publishers.

BUSH, G. W. (2001), “Address to Joint Session of Congress - Transcript”, CCN Website, 21 September. Disponible: http://articles.cnn.com/2001-09-20/us/gen.bush.transcript_1_joint-session-national-anthem-citizens?_s=PM:US Consultado 20/01/2012.

CARPENTER, T. G. (2003). Bad Neighbor Policy: Washington's Futile War on Drugs in Latin America, New York, N.Y.: Palgrave Macmillan.

CHABAT, J. (2010). "La Iniciativa Mérida y la Relación México-Estados Unidos: en busca de la esperanza perdida", Documento de Trabajo 195, CIDE.

CHERNICK, M. C. (2012), Entrevista personal, aprobada por el IRB.

CHRIS K. (2008), "Bush pushes trade pact to aid Colombia and his legacy”, Los Angeles Times, 13/11/2008. 
CNN MÉXICO (2012). "Peña Nieto toma a Colombia como ejemplo para su estrategia de seguridad", Agencia CNN México, 18 de septiembre, disponible en línea: <www.mexico.cnn.com>, consultado en $7 / 11 / 2013$.

CRANDALL, R. (2002). Driven by Drugs: U.S. Policy toward Colombia. Boulder, CO: Lynne Publishers.

CUELlAR, A. (2008). “America's Forgotten War" The Washington Post. 29/10/2008.

ELLIOT, M. (2011). "Viewpoint: how Libya became a French and British war", Time World, 19/03/2011.

GARAY, L. J. y SALCEDO-ALABARÁN, E. (2012). Narcotráfico, Corrupcióny Estados. México: Grijalbo.

GARCÍA, J. (2013). “El infierno se llama Michoacán”, El Mundo, 8/11/2013.

GEORGIOS A.A. (2008). "Colombian Drug Cartels”, en Frank Shanty y Patit Paban Mishra (eds.), Organized Crime: from trafficking to terrorism, Santa Barbara California: ABC-CLIO.

ISACSON, A. (2002). "Extending the War on Terrorism to Colombia: A Bad Idea Whose Time Has Come”. Foreign Policy In Focus, Febrero 6. Disponible en línea: <http://www.fpif.org/articles/ extending_the_war_on_terrorism_to_colombia_a_bad_idea_whose > Consultado en Agosto, 3, 2013.

ISACSON, A. (2002). Don't Call Plan Colombia a Model: On Plan Colombia 1oth Anniverary, Alaims of "Success" Don't Stand Up to Scrutiny, Washington, D.C.: WOLA.

KRAUL, C. (2008), "Bush Pushes Trade Pact to Aid Colombia and his Legacy", Los Angeles Times, Noviembre 13 .

LEE, R. W., y THOUMI, F. E. (2003), “Drugs and Democracy in Colombia”, en Godson, R. (Ed.). Menace to Society: Political-criminal Colllaboration Around the World. Transaction Publishers, pp. 71-97.

LENDMAN, S. (2008). Plan Mexico: Plan Colombia Heads for Mexico. Canada: Global Research Center.

MEARSHEIMER, J. J. (1994), “The false promise of international institutions”, International Security, 19 (3), pp. 5-49.

NADELMANN, E. (2012), "Obama's hypocritical war on marijuana”, The Huffington Post, 25 de mayo. Disponible en línea: <http://www.huffingtonpost.com/ethan-nadelmann/obama-marijuana_b_1546921.html> Consultado en 15/o8/2013. Texto citado traducido del inglés por Roberto Zepeda Martínez.

NADELMANN, E. (2013). "Ending the War on Drugs: Easier Said Than Done", The Huffington Post, 18 de junio. Disponible en línea: <http://www.huffingtonpost.com/ethan-nadelmann/marijuana-legalization-war-on-drugs_b_3456324.html> Consultado en Noviembre, 2, 2013. Texto citado traducido del inglés por Roberto Zepeda Martínez.

NOTIMEX (2013), “Descarta Obama cambiar políticas sobre marihuana”, La Jornada, 22/07/2013.

NURUZZAMAN, M. (2006). "Beyond the Realist Theories: 'Neo-Conservative Realism' and the American Invasion of Iraq" International Studies Perspectives (7), pp. 239-253. 
NYE, J. S. (2004). Soft power: The means to success in world politics. New York: Public Affairs Store.

ORGANIZACIÓN DE ESTADOS AMERICANOS - OEA. (2013). El problema de las drogas en las Américas. Washington D.C. Organización de los Estados Americanos, Secretaría General.

ORGANIZATION OF AMERICAN STATES - OAS. (2013). Report of the Drug Problem in the Americas, Panama: OAS.

PÉREZ, J. C. (2012), “Enrique Peña Nieto: 'La estrategia que se ha seguido contra las drogas en México es equivocada”, La Tercera, 10/06/2012.

REFSHAUGE, E. (2012). "Uribe slams Hugo Chavez during trip to Venezuelan border”, Colombia Reports, Julio, 16. Disponible en línea: <http://colombiareports.co/former-colombian-president-ends-anti-chavez-tour-on-venezuelan-border/>.

RENSSELAER, W. L. III y THOUMI, F. E. (2003). “Drugs and Democracy in Colombia”, en Godson, R. Menace to Society: Political Collaboration around the World, New Brunswick, NJ: Transaction Books.

REUTERS (2013). "Mexico to track drug war victims, compensate families", Chicago Tribute. Enero 9. Disponible: http://www.reuters.com/article/2013/o1/o9/us-mexico-drugs-idUSBRE9081CQ20130109, Consultado en noviembre 2013.

ROSEN, J. (2014). “The Losing War: Plan Colombia and Beyond” Albany, N.Y.: SUNY Press, forthcoming 2014 .

SEELKE, C. R. y FINKLEA, K. M. (2013). "U.S.-Mexican Security Cooperation: The Mérida Initiative and Beyond" .Washington D.C.: Congressional Research Service.

SISTEMA NACIONAL DE SEGURIDAD PÚBLICA (SSN) (2012). Incidencia Delictiva del Fuero Común 2012 y 2013. Secretaria de Gobernación del gobierno federal de México.

THOUMI, F.E. (Ed.). (1995). Political economy and illegal drugs in Colombia (Vol. 2). United United Nations Office on Drugs, \& Crime. (2010). World Drug Report 2010. United Nations Publications.

TOKATLIAN, J.G. (2012) “Drogas: una guerra que fracasó”, La Nación, 13 de marzo. Disponible en línea: <http://www.lanacion.com.ar/1456029-drogas-una-guerra-que-fracaso>, Consultado 12/o7/2013.

UNITED NATIONS OFFICE ON DRUGS AND CRIME - UNODC. (2011). World Drug Report, 2011, New York: UNODC.

UNITED NATIONS OFFICE ON DRUGS AND CRIME - UNODC. (2O13). World Drug Report 2013. United Nations Publications. Disponible en línea: http://www.un.org/, consultado 1/11/2013.

WING, N. y JOHNSON, L. (2013). “Obama Administration Has Spent Nearly \$30o Million Cracking Down On Medical Marijuana: Report”. The Hufftington Post, disponible en línea: $<$ http://www.huffingtonpost.com/2013/o6/13/obama-medical-marijuana_n_3437636.html> Consultado 23/o9/2013. Texto citado traducido del inglés por Roberto Zepeda Martínez. 